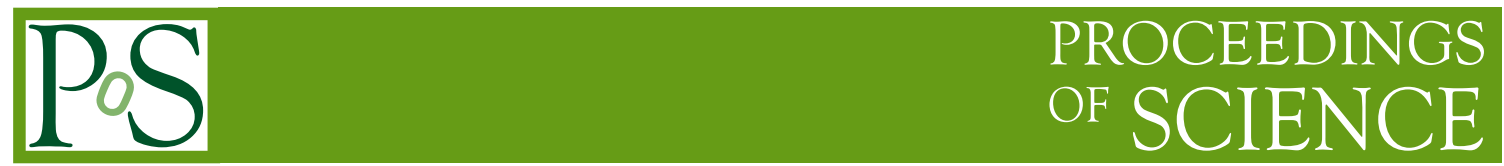

\title{
Global Status of Sterile Neutrino Scenarios
}

\section{Carlo Giunti*}

INFN, Sezione di Torino, Via P. Giuria 1, I-10125 Torino, Italy

E-mail: giunti@to.infn.it

We review the results of the global analyses of short-baseline neutrino oscillation data in $3+1$ and $3+2$ neutrino mixing schemes with one or two light sterile neutrinos. The implications for neutrinoless double- $\beta$ decay are briefly discussed.

XVI International Workshop on Neutrino Telescopes,

2-6 March 2015

Palazzo Franchetti, Istituto Veneto, Venice, Italy

${ }^{*}$ Speaker. 


\section{Introduction}

Neutrino oscillations have been measured with high accuracy in solar, atmospheric and longbaseline neutrino oscillation experiments (see Refs. [1-3]), in agreement with the standard threeneutrino mixing paradigm ( $3 v)$, in which the three active neutrinos $v_{e}, v_{\mu}, v_{\tau}$ are superpositions of three massive neutrinos $v_{1}, v_{2}, v_{3}$ with respective masses $m_{1}, m_{2}, m_{3}$ (see Refs. [4, 5]). There are two independent squared-mass differences, the solar $\Delta m_{\mathrm{SOL}}^{2} \simeq 7.5 \times 10^{-5} \mathrm{eV}^{2}$ and the atmospheric $\Delta m_{\mathrm{ATM}}^{2} \simeq 2.3 \times 10^{-3} \mathrm{eV}^{2}$, which can be interpreted as $\Delta m_{\mathrm{SOL}}^{2}=\Delta m_{21}^{2}$ and $\Delta m_{\mathrm{ATM}}^{2}=\left|\Delta m_{31}^{2}\right| \simeq$ $\left|\Delta m_{32}^{2}\right|$, with $\Delta m_{k j}^{2}=m_{k}^{2}-m_{j}^{2}$.

The completeness of the $3 v$ mixing paradigm has been challenged by the following indications in favor of short-baseline neutrino oscillations, which require the existence of at least one additional squared-mass difference, $\Delta m_{\mathrm{SBL}}^{2}$, which is much larger than $\Delta m_{\mathrm{SOL}}^{2}$ and $\Delta m_{\mathrm{ATM}}^{2}$ :

1. The reactor antineutrino anomaly [6], which is a deficit of the rate of $\bar{v}_{e}$ observed in several short-baseline reactor neutrino experiments in comparison with that expected from a new calculation of the reactor neutrino fluxes $[7,8]$. The statistical significance is about $2.8 \sigma$.

2. The Gallium neutrino anomaly [9-13], consisting in a short-baseline disappearance of $v_{e}$ measured in the Gallium radioactive source experiments GALLEX [14] and SAGE [15] with a statistical significance of about $2.9 \sigma$.

3. The LSND experiment, in which a signal of short-baseline $\bar{v}_{\mu} \rightarrow \bar{v}_{e}$ oscillations has been observed with a statistical significance of about $3.8 \sigma[16,17]$.

In this review, we consider 3+1 [18-21] and 3+2 [22-25] neutrino mixing schemes in which there are one or two additional massive neutrinos at the $\mathrm{eV}$ scale ${ }^{1}$ and the masses of the three standard massive neutrinos are much smaller. Since from the LEP measurement of the invisible width of the $Z$ boson we know that there are only three active neutrinos (see Ref. [4]), in the flavor basis the additional massive neutrinos correspond to sterile neutrinos [31], which do not have standard weak interactions.

The possible existence of sterile neutrinos is very interesting, because they are new particles which could give us precious information on the physics beyond the Standard Model (see Refs. $[32,33])$. The existence of light sterile neutrinos is also very important for astrophysics (see Ref. [34]) and cosmology (see Ref. [35-38]).

In the $3+1$ scheme, the effective probability of $\stackrel{(-)}{v_{\alpha}} \rightarrow \stackrel{(-)}{v_{\beta}}$ transitions in short-baseline experiments has the two-neutrino-like form [19]

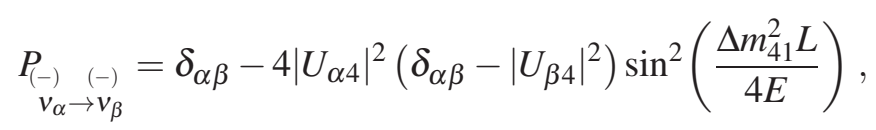

where $U$ is the mixing matrix, $L$ is the source-detector distance, $E$ is the neutrino energy and $\Delta m_{41}^{2}=m_{4}^{2}-m_{1}^{2}=\Delta m_{\mathrm{SBL}}^{2} \sim 1 \mathrm{eV}^{2}$. The electron and muon neutrino and antineutrino appearance and disappearance in short-baseline experiments depend on $\left|U_{e 4}\right|^{2}$ and $\left|U_{\mu 4}\right|^{2}$, which determine the amplitude $\sin ^{2} 2 v_{e \mu}=4\left|U_{e 4}\right|^{2}\left|U_{\mu 4}\right|^{2}$ of $v_{\mu}^{(-)} \rightarrow \stackrel{(-)}{v_{e}}$ transitions, the amplitude $\sin ^{2} 2 v_{e e}=$

\footnotetext{
${ }^{1}$ It is interesting to consider also the $3+1+1$ scheme [26-30], in which one of the two additional massive neutrinos is much heavier than the $\mathrm{eV}$ scale.
} 
$4\left|U_{e 4}\right|^{2}\left(1-\left|U_{e 4}\right|^{2}\right)$ of $v_{e}^{(-)}$disappearance, and the amplitude $\sin ^{2} 2 \vartheta_{\mu \mu}=4\left|U_{\mu 4}\right|^{2}\left(1-\left|U_{\mu 4}\right|^{2}\right)$ of $v_{\mu}^{(-)}$ disappearance.

Since the oscillation probabilities of neutrinos and antineutrinos are related by a complex conjugation of the elements of the mixing matrix (see Ref. [4]), the effective probabilities of shortbaseline $v_{\mu} \rightarrow v_{e}$ and $\bar{v}_{\mu} \rightarrow \bar{v}_{e}$ transitions are equal. Hence, the $3+1$ scheme cannot explain a possible CP-violating difference of $v_{\mu} \rightarrow v_{e}$ and $\bar{v}_{\mu} \rightarrow \bar{v}_{e}$ transitions in short-baseline experiments. In order to allow this possibility, one must consider a $3+2$ scheme, in which, there are four additional effective mixing parameters in short-baseline experiments: $\Delta m_{51}^{2} \geq \Delta m_{41}^{2},\left|U_{e 5}\right|^{2},\left|U_{\mu 5}\right|^{2}$ and $\eta=\arg \left[U_{e 4}^{*} U_{\mu 4} U_{e 5} U_{\mu 5}^{*}\right]$ (see Refs. [5,39]). Since this complex phase appears with different signs in the effective $3+2$ probabilities of short-baseline $v_{\mu} \rightarrow v_{e}$ and $\bar{v}_{\mu} \rightarrow \bar{v}_{e}$ transitions, it can generate measurable $\mathrm{CP}$ violations.

\section{Global fits of short-baseline neutrino oscillations}

Updated global fits of short-baseline neutrino oscillation data have been presented in Refs. $[30,40]$. These analyses take into account the final results of the MiniBooNE experiment, which was made in order to check the LSND signal with about one order of magnitude larger distance $(L)$ and energy $(E)$, but the same order of magnitude for the ratio $L / E$ from which neutrino oscillations depend. Unfortunately, the results of the MiniBooNE experiment are ambiguous, because the LSND signal was not seen in neutrino mode $\left(v_{\mu} \rightarrow v_{e}\right)$ [41] and the $\bar{v}_{\mu} \rightarrow \bar{v}_{e}$ signal observed in 2010 [42] with the first half of the antineutrino data was not observed in the second half of the antineutrino data [43]. Moreover, the MiniBooNE data in both neutrino and antineutrino modes show an excess in the low-energy bins which is widely considered to be anomalous because it is at odds with neutrino oscillations $[44,45]^{2}$.

In the following we summarize the results of an update [48] of the global fit of short-baseline neutrino oscillation data presented in Ref. [30]. We consider the following three groups of experiments:

(A) The $\stackrel{(-)}{v_{\mu}} \rightarrow \stackrel{(-)}{v_{e}}$ appearance data of the LSND [17], MiniBooNE [43], BNL-E776 [49], KARMEN [50], NOMAD [51], ICARUS [52] and OPERA [53] experiments.

(B) The $v_{e}$ disappearance data described in Ref. [13], which take into account the reactor [6-8] and Gallium [9-12,54] anomalies. We extended the data set considered in Ref. [13] by including the Daya Bay measurement of the reactor electron antineutrino flux at the near detector [55] and the results of the Chooz [56], Palo Verde [57] and Double Chooz [58,59] reactor experiments, as suggested in Ref. [60].

(C) The constraints on $v_{\mu}$ disappearance obtained from the data of the CDHSW experiment [61], from the analysis [24] of the data of atmospheric neutrino oscillation experiments ${ }^{3}$, from the

\footnotetext{
${ }^{2}$ The interesting possibility of reconciling the low-energy anomalous data with neutrino oscillations through energy reconstruction effects proposed in Ref. [46,47] still needs a detailed study.

${ }^{3}$ The IceCube data, which could give a marginal contribution [62,63], have not been considered because the analysis is too complicated and subject to large uncertainties.
} 


\begin{tabular}{c|cccc|cc} 
& $3+1$ & $3+1$ & $3+1$ & $3+1$ & $3+2$ & $3+2$ \\
& LOW & HIG & noMB & noLSND & LOW & HIG \\
\hline$\chi_{\min }^{2}$ & 306.0 & 276.3 & 251.2 & 291.3 & 299.6 & 271.1 \\
$\mathrm{NDF}$ & 268 & 262 & 230 & 264 & 264 & 258 \\
$\mathrm{GoF}$ & $5 \%$ & $26 \%$ & $16 \%$ & $12 \%$ & $7 \%$ & $28 \%$ \\
\hline$\left(\chi_{\min }^{2}\right)_{\mathrm{APP}}$ & 98.9 & 77.0 & 50.9 & 91.8 & 86.0 & 69.6 \\
$\left(\chi_{\min }^{2}\right)_{\mathrm{DIS}}$ & 194.4 & 194.4 & 194.4 & 194.4 & 192.9 & 192.9 \\
$\Delta \chi_{\mathrm{PG}}^{2}$ & 13.0 & 5.3 & 6.2 & 5.3 & 20.7 & 8.6 \\
$\mathrm{NDF}_{\mathrm{PG}}$ & 2 & 2 & 2 & 2 & 4 & 4 \\
$\mathrm{GoF}_{\mathrm{PG}}$ & $0.1 \%$ & $7 \%$ & $5 \%$ & $7 \%$ & $0.04 \%$ & $7 \%$ \\
\hline$\Delta \chi_{\mathrm{NO}}^{2}$ & 49.2 & 47.7 & 48.1 & 11.4 & 55.7 & 52.9 \\
$\mathrm{NDF}_{\mathrm{NO}}$ & 3 & 3 & 3 & 3 & 7 & 7 \\
$n \sigma_{\mathrm{NO}}$ & $6.4 \sigma$ & $6.3 \sigma$ & $6.4 \sigma$ & $2.6 \sigma$ & $6.1 \sigma$ & $5.9 \sigma$
\end{tabular}

Table 1: Results of the fit of short-baseline data $[30,48]$ taking into account all MiniBooNE data (LOW), only the MiniBooNE data above $475 \mathrm{MeV}$ (HIG), without MiniBooNE data (noMB) and without LSND data (noLSND) in the $3+1$ and $3+2$ schemes. The first three lines give the minimum $\chi^{2}\left(\chi_{\min }^{2}\right)$, the number of degrees of freedom (NDF) and the goodness-of-fit (GoF). The following five lines give the quantities relevant for the appearance-disappearance (APP-DIS) parameter goodness-of-fit (PG) [67]. The last three lines give the difference between the $\chi^{2}$ without short-baseline oscillations and $\chi_{\min }^{2}\left(\Delta \chi_{\mathrm{NO}}^{2}\right)$, the corresponding difference of number of degrees of freedom $\left(\mathrm{NDF}_{\mathrm{NO}}\right)$ and the resulting number of $\sigma$ 's $\left(n \sigma_{\mathrm{NO}}\right)$ for which the absence of oscillations is disfavored.

analysis [44] of the MINOS neutral-current data [64] and from the analysis of the SciBooNEMiniBooNE neutrino [65] and antineutrino [66] data.

Table 1 summarizes the statistical results obtained from global fits of the data above in the $3+1$ and $3+2$ schemes $[30,48]$. In the LOW fits all the MiniBooNE data are considered, including the anomalous low-energy bins, which are omitted in the HIG fits. There is also a 3+1-noMB fit without MiniBooNE data and a 3+1-noLSND fit without LSND data.

From Tab.1, one can see that in all fits which include the LSND data the absence of shortbaseline oscillations is disfavored by about $6 \sigma$, because the improvement of the $\chi^{2}$ with shortbaseline oscillations is much larger than the number of oscillation parameters.

In all the 3+1 and 3+2 schemes the goodness-of-fit in the LOW analysis is significantly worse than that in the HIG analysis and the appearance-disappearance parameter goodness-of-fit is much worse. This result confirms the fact that the MiniBooNE low-energy anomaly is incompatible with neutrino oscillations, because it would require a small value of $\Delta m_{41}^{2}$ and a large value of $\sin ^{2} 2 \vartheta_{e \mu}$ [44,45], which are excluded by the data of other experiments (see Ref. [30] for further details $)^{4}$. Note that the appearance-disappearance tension in the $3+2$-LOW fit is even worse than that in the $3+1-\mathrm{LOW}$ fit, since the $\Delta \chi_{\mathrm{PG}}^{2}$ is so much larger that it cannot be compensated by the

\footnotetext{
${ }^{4}$ One could fit the three anomalous MiniBooNE low-energy bins in a 3+2 scheme [39] by considering the appearance data without the ICARUS [52] and OPERA [53] constraints, but the required large transition probability is excluded by the disappearance data.
} 

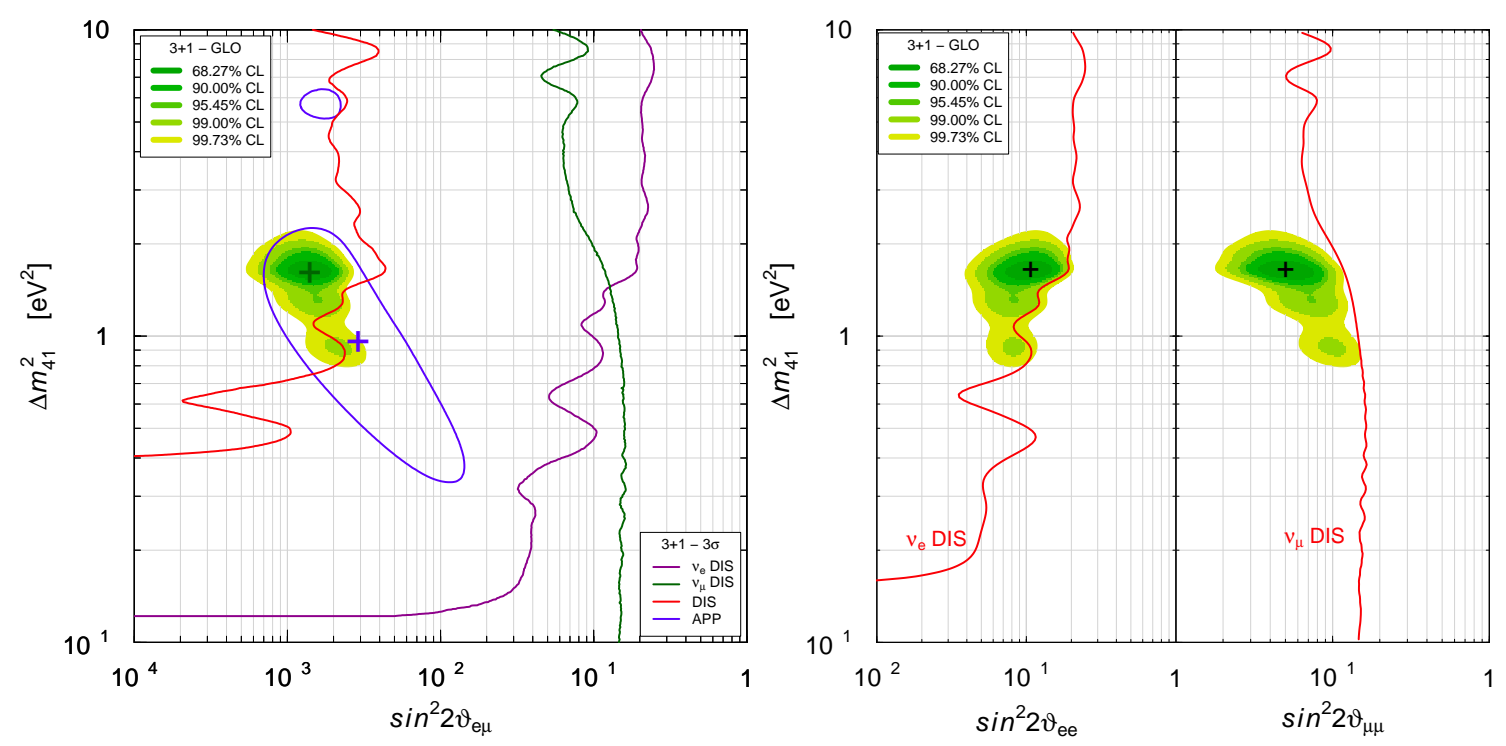

Figure 1: Allowed regions in the $\sin ^{2} 2 \vartheta_{e \mu}-\Delta m_{41}^{2}, \sin ^{2} 2 \vartheta_{e e}-\Delta m_{41}^{2}$ and $\sin ^{2} 2 \vartheta_{\mu \mu}-\Delta m_{41}^{2}$ planes obtained in the global (GLO) 3+1-HIG fit [30,48] of short-baseline neutrino oscillation data compared with the $3 \sigma$ allowed regions obtained from $v_{\mu} \rightarrow \stackrel{(-)}{v_{e}}$ short-baseline appearance data (APP) and the $3 \sigma$ constraints obtained from $\stackrel{(-)}{v}_{e}$ short-baseline disappearance data $\left(v_{e}\right.$ DIS $), \stackrel{(-)}{v_{\mu}}$ short-baseline disappearance data $\left(v_{\mu}\right.$ DIS) and the combined short-baseline disappearance data (DIS). The best-fit points of the GLO and APP fits are indicated by crosses.

additional degrees of freedom ${ }^{5}$. Therefore, we think that it is very likely that the MiniBooNE lowenergy anomaly has an explanation which is different from neutrino oscillations and the HIG fits are more reliable than the LOW fits.

The 3+2 mixing scheme was considered to be interesting in 2010 when the MiniBooNE neutrino [41] and antineutrino [42] data showed a CP-violating tension, but this tension almost disappeared in the final MiniBooNE data [43]. In fact, from Tab.1 one can see that there is little improvement of the $3+2-\mathrm{HIG}$ fit with respect to the $3+1-\mathrm{HIG}$ fit, in spite of the four additional parameters and the additional possibility of $\mathrm{CP}$ violation. Moreover, since the $\mathrm{p}$-value obtained by restricting the $3+2$ scheme to $3+1$ disfavors the $3+1$ scheme only at $1.1 \sigma[30,48]$, we think that considering the larger complexity of the $3+2$ scheme is not justified by the data ${ }^{6}$.

Figure 1 shows the allowed regions in the $\sin ^{2} 2 \vartheta_{e \mu}-\Delta m_{41}^{2}, \sin ^{2} 2 \vartheta_{e e}-\Delta m_{41}^{2}$ and $\sin ^{2} 2 \vartheta_{\mu \mu}-$ $\Delta m_{41}^{2}$ planes obtained in the 3+1-HIG fit of Ref. [30,48]. These regions are relevant, respectively, for $\stackrel{(-)}{v_{\mu}} \rightarrow \stackrel{(-)}{v_{e}}$ appearance, $\stackrel{(-)}{v}_{e}$ disappearance and $\stackrel{(-)}{v_{\mu}}$ disappearance searches. The corresponding marginal allowed intervals of the oscillation parameters are given in Tab.2. Figure 1 shows also the region allowed by $\stackrel{(-)}{v_{\mu}} \rightarrow \stackrel{(-)}{v_{e}}$ appearance data and the constraints from $\stackrel{(-)}{v_{e}}$ disappearance and $\stackrel{(-)}{v_{\mu}}$ disappearance data. One can see that the combined disappearance constraint in the $\sin ^{2} 2 \vartheta_{e \mu}-$ $\Delta m_{41}^{2}$ plane excludes a large part of the region allowed by $\stackrel{(-)}{v_{\mu}} \rightarrow \stackrel{(-)}{v_{e}}$ appearance data, leading to the well-known appearance-disappearance tension [39, 40, 44, 45, 68-71] quantified by the parameter

\footnotetext{
${ }^{5}$ This behavior has been explained in Ref. [68]. It was found also in the analysis presented in Ref. [40].

${ }^{6}$ See however the somewhat different conclusions reached in Ref. [40].
} 


\begin{tabular}{c|cccc}
$\mathrm{CL}$ & $\Delta m_{41}^{2}\left[\mathrm{eV}^{2}\right]$ & $\sin ^{2} 2 \vartheta_{e \mu}$ & $\sin ^{2} 2 \vartheta_{e e}$ & $\sin ^{2} 2 \vartheta_{\mu \mu}$ \\
\hline $68.27 \%$ & $1.57-1.72$ & $0.0011-0.0018$ & $0.085-0.13$ & $0.039-0.066$ \\
\hline $90.00 \%$ & $1.53-1.78$ & $0.00098-0.002$ & $0.071-0.15$ & $0.032-0.078$ \\
\hline $95.00 \%$ & $1.50-1.84$ & $0.00-0.00$ & $0.065-0.16$ & $0.03-0.083$ \\
\hline $95.45 \%$ & $1.50-1.84$ & $0.00089-0.0021$ & $0.063-0.16$ & $0.03-0.085$ \\
\hline $99.00 \%$ & $1.24-1.95$ & $0.00074-0.0023$ & $0.054-0.18$ & $0.025-0.095$ \\
\hline $99.73 \%$ & $0.87-2.04$ & $0.00065-0.0026$ & $0.046-0.19$ & $0.021-0.12$
\end{tabular}

Table 2: Marginal allowed intervals of the oscillation parameters obtained in the global 3+1-HIG fit of short-baseline neutrino oscillation data [30,48].

goodness-of-fit in Tab.1.

It is interesting to investigate what is the impact of the MiniBooNE experiment on the global analysis of short-baseline neutrino oscillation data. With this aim, we consider two additional $3+1$ fits [30,48]: a 3+1-noMB fit without MiniBooNE data and a 3+1-noLSND fit without LSND data. From Tab. 1 one can see that the results of the $3+1$-noMB fit are similar to those of the $3+1-$ HIG fit and the exclusion of the case of no-oscillations remains at the level of $6 \sigma$. On the other hand, in the 3+1-noLSND fit, without LSND data, the exclusion of the case of no-oscillations drops dramatically to $2.6 \sigma$. In fact, in this case the main indication in favor of short-baseline oscillations is given by the reactor and Gallium anomalies which have a similar statistical significance [13]. Therefore, it is clear that the LSND experiment is still crucial for the indication in favor of shortbaseline $\bar{v}_{\mu} \rightarrow \bar{v}_{e}$ transitions and the MiniBooNE experiment has been rather inconclusive.

\section{Neutrinoless double- $\beta$ decay}

The existence of massive neutrinos at the eV scale can be probed in $\beta$-decay experiments [73$79]$ and in neutrinoless double- $\beta$ decay experiments $[13,72,80-88]$. Here we discuss briefly only neutrinoless double- $\beta$ decay, which in the case of $3+1$ mixing depends on the effective Majorana mass

$$
\left|m_{\beta \beta}\right|=\left.|| U_{e 1}\right|^{2} m_{1}+\left|U_{e 2}\right|^{2} e^{i \alpha_{2}} m_{2}+\left|U_{e 3}\right|^{2} e^{i \alpha_{3}} m_{3}+\left|U_{e 4}\right|^{2} e^{i \alpha_{4}} m_{4} \mid,
$$

with three completely unknown complex phases $\alpha_{2}, \alpha_{3}, \alpha_{4}$ which can generate cancellations between the different mass contributions. Figure 2 shows the range of allowed values of $\left|m_{\beta \beta}\right|$ as a function of the lightest neutrino mass in the cases of $3 v$ and $3+1$ mixing with Normal and Inverted Ordering of the three lightest neutrinos [72]. The $3 v$ mixing parameters have been taken from Ref. [89] and the sterile neutrino mixing is that obtained in the 3+1-HIG global fit of short-baseline neutrino oscillation data discussed in Section 2.

From Fig.2 one can see that the presence of an additional massive neutrinos at the eV scale can change dramatically the predictions for the possible range of values of $\left|m_{\beta \beta}\right|[13,72,80-88]$.

\section{Conclusions}

In conclusion, the results of the global fit of short-baseline neutrino oscillation data $[30,48]$ show that the data can be explained by $3+1$ neutrino mixing and this simplest scheme beyond 

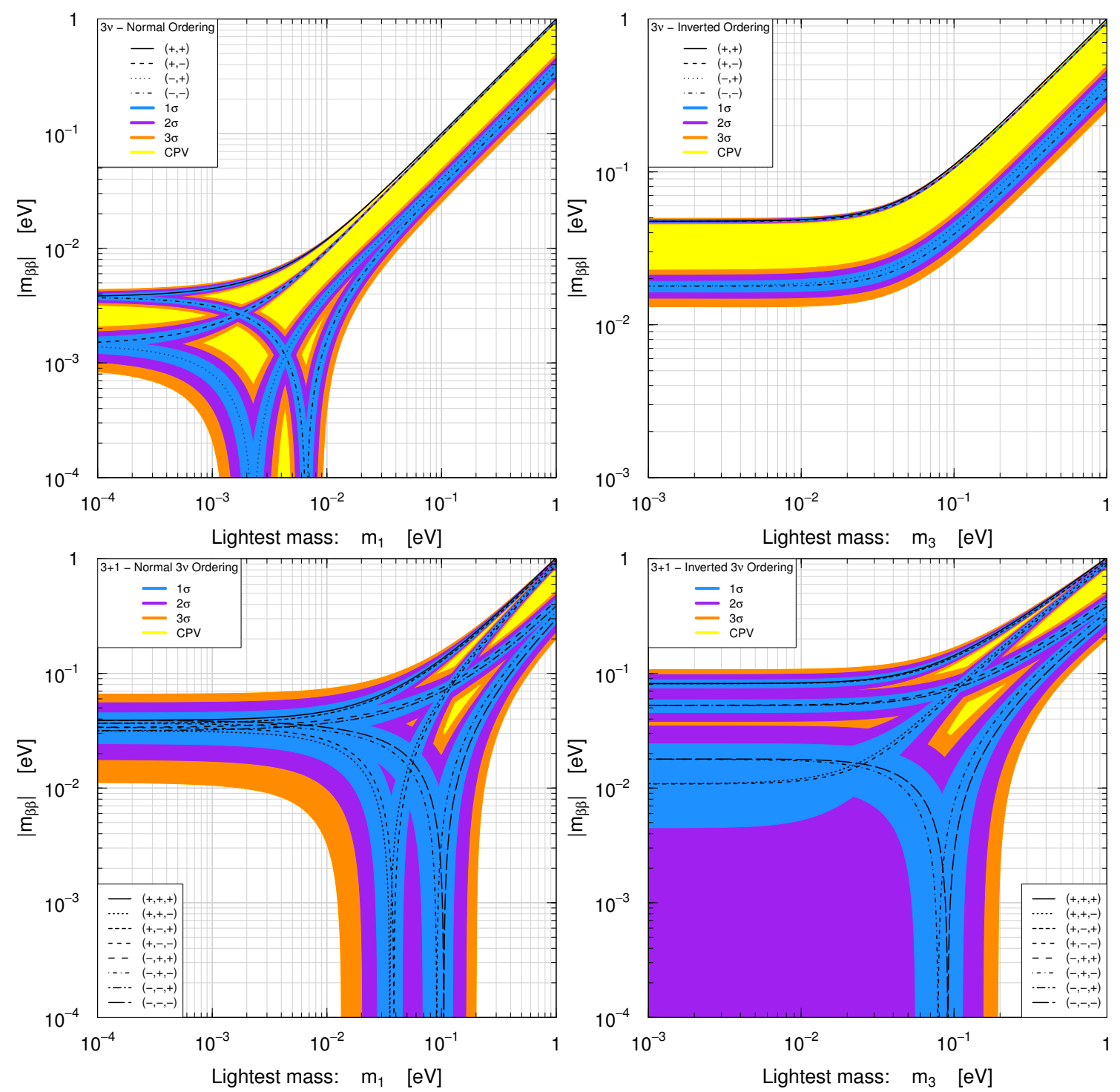

Figure 2: Value of the effective Majorana mass $\left|m_{\beta \beta}\right|$ as a function of the lightest neutrino mass in the cases of $3 v$ and 3+1 mixing with Normal and Inverted Ordering of the three lightest neutrinos [72]. The signs in the legends indicate the signs of $e^{i \alpha_{2}}, e^{i \alpha_{3}}, e^{i \alpha_{4}}= \pm 1$ for the cases in which CP is conserved. The intermediate yellow regions are allowed only in the case of $\mathrm{CP}$ violation.

three-neutrino mixing cannot be rejected in favor of the more complex $3+2$ scheme. The lowenergy MiniBooNE anomaly cannot be explained by neutrino oscillations in any of these schemes. Moreover, the only substantial indication in favor of short-baseline $\bar{v}_{\mu} \rightarrow \bar{v}_{e}$ appearance is still given by the old LSND data and the MiniBooNE experiment has been inconclusive. Hence new experiments are needed in order to check this signal [90-98].

\section{References}

[1] D. Forero, M. Tortola and J. Valle, Phys. Rev. D86 (2012) 073012, arXiv:1205.4018. 
[2] G. Fogli et al., Phys. Rev. D86 (2012) 013012, arXiv:1205.5254.

[3] M. Gonzalez-Garcia et al., JHEP 12 (2012) 123, arXiv:1209.3023.

[4] C. Giunti and C.W. Kim, Fundamentals of Neutrino Physics and Astrophysics (Oxford University Press, Oxford, UK, 2007).

[5] M.C. Gonzalez-Garcia and M. Maltoni, Phys. Rept. 460 (2008) 1, arXiv:0704.1800.

[6] G. Mention et al., Phys. Rev. D83 (2011) 073006, arXiv:1101.2755.

[7] T.A. Mueller et al., Phys. Rev. C83 (2011) 054615, arXiv:1101.2663.

[8] P. Huber, Phys. Rev. C84 (2011) 024617, arXiv:1106.0687.

[9] SAGE, J.N. Abdurashitov et al., Phys. Rev. C73 (2006) 045805, nucl-ex/0512041.

[10] M. Laveder, Nucl. Phys. Proc. Suppl. 168 (2007) 344.

[11] C. Giunti and M. Laveder, Mod. Phys. Lett. A22 (2007) 2499, hep-ph/0610352.

[12] C. Giunti and M. Laveder, Phys. Rev. C83 (2011) 065504, arXiv:1006.3244.

[13] C. Giunti et al., Phys. Rev. D86 (2012) 113014, arXiv:1210.5715.

[14] F. Kaether et al., Phys. Lett. B685 (2010) 47, arXiv:1001.2731.

[15] SAGE, J.N. Abdurashitov et al., Phys. Rev. C80 (2009) 015807, arXiv:0901.2200.

[16] LSND, C. Athanassopoulos et al., Phys. Rev. Lett. 75 (1995) 2650, nucl-ex/9504002.

[17] LSND, A. Aguilar et al., Phys. Rev. D64 (2001) 112007, hep-ex/0104049.

[18] N. Okada and O. Yasuda, Int. J. Mod. Phys. A12 (1997) 3669, hep-ph/9606411.

[19] S.M. Bilenky, C. Giunti and W. Grimus, Eur. Phys. J. C1 (1998) 247, hep-ph/9607372.

[20] S.M. Bilenky et al., Phys. Rev. D60 (1999) 073007, hep-ph/9903454.

[21] M. Maltoni et al., New J. Phys. 6 (2004) 122, hep-ph/0405172.

[22] M. Sorel, J. Conrad and M. Shaevitz, Phys. Rev. D70 (2004) 073004, hep-ph/0305255.

[23] G. Karagiorgi et al., Phys. Rev. D75 (2007) 013011, hep-ph/0609177.

[24] M. Maltoni and T. Schwetz, Phys. Rev. D76 (2007) 093005, arXiv:0705.0107.

[25] G. Karagiorgi et al., Phys. Rev. D80 (2009) 073001, arXiv:0906.1997.

[26] A.E. Nelson, Phys. Rev. D84 (2011) 053001, arXiv:1010.3970.

[27] J. Fan and P. Langacker, JHEP 04 (2012) 083, arXiv:1201.6662.

[28] E. Kuflik, S.D. McDermott and K.M. Zurek, Phys. Rev. D86 (2012) 033015, arXiv:1205.1791.

[29] J. Huang and A.E. Nelson, Phys.Rev. D88 (2013) 033016, arXiv:1306.6079.

[30] C. Giunti et al., Phys.Rev. D88 (2013) 073008, arXiv:1308.5288.

[31] B. Pontecorvo, Sov. Phys. JETP 26 (1968) 984.

[32] R.R. Volkas, Prog. Part. Nucl. Phys. 48 (2002) 161, hep-ph/0111326.

[33] R.N. Mohapatra and A.Y. Smirnov, Ann. Rev. Nucl. Part. Sci. 56 (2006) 569, hep-ph/0603118.

[34] A. Diaferio and G.W. Angus, arXiv:1206.6231. 
[35] J. Lesgourgues et al., Neutrino Cosmology (Cambridge University Press, 2013).

[36] S. Riemer-Sorensen, D. Parkinson and T.M. Davis, Publ.Astron.Soc.Austral. 30 (2013) e029, arXiv:1301.7102.

[37] M. Archidiacono et al., Adv.High Energy Phys. 2013 (2013) 191047, arXiv:1307.0637.

[38] J. Lesgourgues and S. Pastor, New J.Phys. 16 (2014) 065002, arXiv:1404.1740.

[39] J. Conrad et al., Adv.High Energy Phys. 2013 (2013) 163897, arXiv:1207.4765.

[40] J. Kopp et al., JHEP 1305 (2013) 050, arXiv:1303.3011.

[41] MiniBooNE, A.A. Aguilar-Arevalo et al., Phys. Rev. Lett. 102 (2009) 101802, arXiv:0812.2243.

[42] MiniBooNE, A.A. Aguilar-Arevalo et al., Phys. Rev. Lett. 105 (2010) 181801, arXiv:1007.1150.

[43] MiniBooNE, A. Aguilar-Arevalo et al., Phys.Rev.Lett. 110 (2013) 161801, arXiv:1303.2588.

[44] C. Giunti and M. Laveder, Phys.Rev. D84 (2011) 093006, arXiv:1109.4033.

[45] C. Giunti and M. Laveder, Phys. Lett. B706 (2011) 200, arXiv:1111.1069.

[46] M. Martini, M. Ericson and G. Chanfray, Phys. Rev. D85 (2012) 093012, arXiv:1202.4745.

[47] M. Martini, M. Ericson and G. Chanfray, Phys. Rev. D87 (2013) 013009, arXiv:1211.1523.

[48] S. Gariazzo et al., (2015), in preparation.

[49] BNL-E776, L. Borodovsky et al., Phys. Rev. Lett. 68 (1992) 274.

[50] KARMEN, B. Armbruster et al., Phys. Rev. D65 (2002) 112001, hep-ex/0203021.

[51] NOMAD, P. Astier et al., Phys. Lett. B570 (2003) 19, hep-ex/0306037.

[52] ICARUS, M. Antonello et al., Eur.Phys.J. C73 (2013) 2599, arXiv:1307.4699.

[53] OPERA, N. Agafonova et al., JHEP 1307 (2013) 004, arXiv:1303.3953.

[54] M.A. Acero, C. Giunti and M. Laveder, Phys. Rev. D78 (2008) 073009, arXiv:0711.4222.

[55] C. Zhang, (2014), Talk presented at Neutrino 2014, XXVI International Conference on Neutrino Physics and Astrophysics, 2-7 June 2014, Boston, Massachusetts, USA.

[56] CHOOZ, M. Apollonio et al., Eur. Phys. J. C27 (2003) 331, hep-ex/0301017.

[57] Palo Verde, F. Boehm et al., Phys. Rev. D64 (2001) 112001, hep-ex/0107009.

[58] Double Chooz, Y. Abe et al., Phys. Rev. D86 (2012) 052008, arXiv:1207.6632.

[59] Double Chooz, Y. Abe et al., Phys.Lett. B723 (2013) 66, arXiv:1301.2948.

[60] C. Zhang, X. Qian and P. Vogel, Phys.Rev. D87 (2013) 073018, arXiv:1303.0900.

[61] CDHSW, F. Dydak et al., Phys. Lett. B134 (1984) 281.

[62] A. Esmaili, F. Halzen and O.L.G. Peres, JCAP 1211 (2012) 041, arXiv:1206.6903.

[63] A. Esmaili and A.Y. Smirnov, JHEP 1312 (2013) 014, arXiv:1307.6824.

[64] MINOS, P. Adamson et al., Phys. Rev. Lett. 107 (2011) 011802, arXiv:1104.3922.

[65] SciBooNE-MiniBooNE, K.B.M. Mahn et al., Phys. Rev. D85 (2012) 032007, arXiv:1106.5685.

[66] SciBooNE-MiniBooNE, G. Cheng et al., Phys. Rev. D86 (2012) 052009, arXiv:1208.0322. 
[67] M. Maltoni and T. Schwetz, Phys. Rev. D68 (2003) 033020, hep-ph/0304176.

[68] M. Archidiacono et al., Phys.Rev. D87 (2013) 125034, arXiv:1302.6720.

[69] J. Kopp, M. Maltoni and T. Schwetz, Phys. Rev. Lett. 107 (2011) 091801, arXiv:1103.4570.

[70] C. Giunti and M. Laveder, Phys.Rev. D84 (2011) 073008, arXiv:1107.1452.

[71] M. Archidiacono et al., Phys. Rev. D86 (2012) 065028, arXiv:1207.6515.

[72] C. Giunti and E.M. Zavanin, arXiv:1505.00978.

[73] C. Kraus et al., Eur.Phys.J. C73 (2013) 2323, arXiv:1210.4194.

[74] A. Belesev et al., JETP Lett. 97 (2013) 67, arXiv:1211.7193.

[75] A. Belesev et al., J.Phys. G41 (2014) 015001, arXiv:1307.5687.

[76] A.S. Riis and S. Hannestad, JCAP 1102 (2011) 011, arXiv:1008.1495.

[77] J.A. Formaggio and J. Barrett, Phys. Lett. B706 (2011) 68, arXiv:1105.1326.

[78] A.S. Riis, S. Hannestad and C. Weinheimer, Phys. Rev. C84 (2011) 045503, arXiv:1105.6005.

[79] A. Esmaili and O.L.G. Peres, Phys. Rev. D85 (2012) 117301, arXiv:1203.2632.

[80] S. Goswami and W. Rodejohann, Phys. Rev. D73 (2006) 113003, hep-ph/0512234.

[81] S. Goswami and W. Rodejohann, JHEP 10 (2007) 073, arXiv:0706.1462.

[82] J. Barry, W. Rodejohann and H. Zhang, JHEP 07 (2011) 091, arXiv:1105.3911.

[83] Y. Li and S. Liu, Phys. Lett. B706 (2012) 406, arXiv:1110.5795.

[84] W. Rodejohann, J. Phys. G39 (2012) 124008, arXiv:1206.2560.

[85] I. Girardi, A. Meroni and S.T. Petcov, JHEP 1311 (2013) 146, arXiv:1308.5802.

[86] S. Pascoli, M. Mitra and S. Wong, Phys.Rev. D90 (2014) 093005, arXiv:1310.6218.

[87] A. Meroni and E. Peinado, Phys.Rev. D90 (2014) 053002, arXiv:1406.3990.

[88] A. Abada, V.D. Romeri and A. Teixeira, JHEP 1409 (2014) 074, arXiv:1406.6978.

[89] F. Capozzi et al., Phys.Rev. D89 (2014) 093018, arXiv:1312.2878.

[90] K.N. Abazajian et al., arXiv:1204.5379.

[91] C. Rubbia et al., arXiv:1304.2047.

[92] OscSNS, M. Elnimr et al., arXiv:1307.7097.

[93] J.P. Delahaye et al., arXiv:1308.0494.

[94] LArTPC, B. Fleming, O. Palamara and . D.Schmitz, arXiv:1309.7987.

[95] M. Antonello et al., arXiv:1312.7252.

[96] nuSTORM, D. Adey et al., Phys.Rev.D (2014), arXiv:1402.5250.

[97] C. Rubbia, arXiv:1408.6431.

[98] MicroBooNE, LAr1-ND, ICARUS-WA104, R. Acciarri et al., arXiv:1503.01520. 